Port Acadie

Revue interdisciplinaire en études acadiennes

An Interdisciplinary Review in Acadian Studies

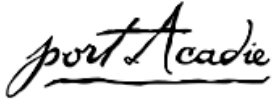

\title{
Antonine Maillet : conteuse de l'Acadie? ou la question du positionnement de l'écrivaine
}

\section{Nicolas Nicaise}

Numéro 22-23, automne 2012, printemps 2013

URI : https://id.erudit.org/iderudit/1014981ar

DOI : https://doi.org/10.7202/1014981ar

Aller au sommaire du numéro

\section{Éditeur(s)}

Université Sainte-Anne

ISSN

1498-7651 (imprimé)

1916-7334 (numérique)

Découvrir la revue

Citer cet article

Nicaise, N. (2012). Antonine Maillet : conteuse de l'Acadie? ou la question du positionnement de l'écrivaine. Port Acadie, (22-23), 183-200.

https://doi.org/10.7202/1014981ar

\section{Résumé de l'article}

Par le biais de l'analyse du discours, nous proposons d'étudier comment Antonine Maillet a inscrit la figure de conteuse au sein de certains de ses textes et dans les propos qu'elle a tenus sur ceux-ci. En observant également le relais que cette figure a trouvé dans le discours de la critique, nous envisageons tout au long de cet article ce positionnement et la vision qu'il donne de l'entreprise de l'écrivaine. Plus généralement, il s'agit d'analyser un faisceau cohérent de discours (littéraire et métalittéraire) et l'impact de celui-ci sur la réception de l'oeuvre mailletienne en fonction des sphères littéraires dans lesquelles ces différents types de discours s'inscrivent. 


\title{
Antonine Maillet : conteuse de l'Acadie? ou la question du positionnement de l'écrivaine
}

\author{
Nicolas Nicaise \\ Université de Moncton - \\ Université de Liège
}

\begin{abstract}
Résumé
Par le biais de l'analyse du discours, nous proposons d'étudier comment Antonine Maillet a inscrit la figure de conteuse au sein de certains de ses textes et dans les propos qu'elle a tenus sur ceux-ci. En observant également le relais que cette figure a trouvé dans le discours de la critique, nous envisageons tout au long de cet article ce positionnement et la vision qu'il donne de l'entreprise de l'écrivaine. Plus généralement, il s'agit d'analyser un faisceau cohérent de discours (littéraire et métalittéraire) et l'impact de celui-ci sur la réception de l'œuvre mailletienne en fonction des sphères littéraires dans lesquelles ces différents types de discours s’inscrivent.
\end{abstract}

L'intérêt de la critique pour l'œuvre d'Antonine Maillet a ponctué le parcours de l'auteur depuis ses débuts, mais s'est intensifié avec la publication de La Sagouine ${ }^{1}$ (1971) et de Pélagie-la-Charette ${ }^{2}$ (prix Goncourt, 1979). Ces écrits lui ont en effet octroyé une reconnaissance et une visibilité internationales. Le colloque de $1988^{3}$, organisé à l'Université de Moncton et consacré à cet auteur, a permis d'envisager plus largement la réception plurielle dont ses œuvres ont fait l'objet. En nous fondant sur les perspectives fournies par ce colloque, nous voudrions, dans cet article, jeter un regard particulier sur le discours critique associé aux textes de Maillet en abordant une thématique particulière, celle du statut de l'écrivaine.

Notre hypothèse sera de considérer ce statut ambigu de Maillet oscillant entre écrivaine et conteuse - accordé volontiers par les critiques et revendiqué par l'auteur - comme révélateur d'une tension propre à l'écrivaine et à son œuvre. La question du rapport de l'auteur à un genre (le conte) et à une langue (dont l'oscillation entre "oralité " et "littérarité " est constante) sera dès lors au centre de notre démarche. Pour rendre compte des enjeux liés à ce positionnement, nous mobiliserons certains

1. Antonine Maillet, La Sagouine - Pièce pour une femme seule, Montréal, Leméac, 1971.

2. Antonine Maillet, Pélagie-la-Charrette, Paris, Grasset, 1979.

3. Les actes de ce colloque ont été publiés comme suit : Marguerite Maillet et Judith Hamel (dir.), La Réception des œuvres d'Antonine Maillet - Actes du colloque international organisé par la Chaire d'études acadiennes les 13, 14 et 15 octobre 1988, Moncton, Chaire d'études acadiennes, 1989. 
des concepts proposés par Dominique Maingueneau - dans Le Discours littéraire 4 - afin d'envisager certaines réflexions sur l'institution littéraire.

\section{Une approche théorique spécifique}

L'idée maîtresse de notre approche est de considérer la problématique du statut de l'écrivaine en tant que positionnement au sein de l'institution littéraire. Puisque "l'institution littéraire elle-même est sans cesse reconfigurée par les discours qu'elle rend possible $»^{5}$ et que la question du statut de Maillet est récurrente dans ceux-ci, la perspective que nous adopterons consiste à étudier ce que l'on peut nommer avec Maingueneau "l'institution discursive », c'est-à-dire le lieu d'articulation entre d'une part les institutions vues comme les cadres qui sous-tendent l'énonciation et d'autre part «le mouvement par lequel s'institue le discours $\|^{6}$.

Mais cette tension paradoxale renvoie aussi à la position spécifique de l'écrivain que Maingueneau érige en concept : la "paratopie ». Il définit celui-ci comme " une localité paradoxale [...] qui n'est pas l'absence de tout lieu, mais une difficile négociation entre le lieu et le non-lieu, une localisation parasitaire, qui vit de l'impossibilité même de se stabiliser " ${ }^{7}$. Cette notion questionne l'inscription elle-même de l'écrivain dans l'univers social et littéraire qui passe notamment par des procédés de légitimation. Maingueneau les place d'ailleurs au cœur de l'analyse du discours :

\footnotetext{
l'analyse du discours [...] doit s'attacher à montrer la connexité de l'intradiscursif et de l'extradiscursif, l'intrication d'une organisation textuelle et d'une activité énonciative. Leur énonciation se déploie comme dispositif de légitimation de son propre espace, y compris sur son versant institutionnel, elle articule l'engendrement d'un texte et une manière de s'inscrire dans un univers social. ${ }^{8}$
}

L'écrivain en quête de reconnaissance adopte en effet différentes stratégies pour se légitimer dont la position qu'il se propose d'assumer fait partie. Le statut ambigu de Maillet révélé par le discours critique renvoie directement, d'après nous, à cette « activité énonciative » qui est non seulement présente dans le texte mailletien, mais également en dehors de lui.

4. Dominique Maingueneau, Le Discours littéraire - Paratopie et scène d'énonciation, Paris, Armand Colin, 2004.

5. Id., p. 42.

6. Id., p. 42 .

7. Id., p. 52-53.

8. Id., p. 48 . 
L'énonciation de l'écrivaine est étroitement liée au champ littéraire auquel elle appartient. Or le champ littéraire acadien commence seulement à s'institutionnaliser avec Antonine Maillet notamment car, jusque dans les années cinquante, celui-ci est "très réduit (essais historiques, romans de la mémoire) [et] le débat politique encore soumis aux derniers diktats de l'élite religieuse " 9 .

Le degré d'institutionnalisation varie donc en fonction de l'ensemble littéraire observé et n'est pas sans influence sur les relations ou rapports de force qu'entretiennent les différents ensembles littéraires francophones. Benoît Denis et Jean-Marie Klinkenberg ${ }^{10}$ considèrent d'ailleurs - au travers de leur système gravitationnel - deux types de forces qui régissent, selon eux, les rapports entre ensembles littéraires : les forces centrifuges et les forces centripètes. Ce sont justement ces forces qui induisent certaines stratégies spécifiques chez les écrivains périphériques. Pascale Casanova identifie à ce sujet "deux grandes "familles" de stratégies [...] à l'intérieur des espaces littéraires [...] d'une part l'assimilation, [...] et d'autre part la différenciation $»^{11}$ au travers desquelles l'écrivain vise une certaine reconnaissance.

Ces deux grandes tendances peuvent également se superposer chez un même écrivain. Dans le cas de Maillet, par exemple, le recours à Rabelais (non seulement par une intertextualité riche, mais aussi par l'expression d'une certaine idée de la langue) apparaît comme une de ces stratégies d'assimilation, Rabelais étant un auteur canonique de la littérature française. Mais à côté de cette dimension fortement littéraire, le positionnement de l'auteur comme conteuse, pour prendre un autre exemple, est plutôt de l'ordre de la différenciation. Le statut de conteuse n'a en effet pas le même prestige et ne recouvre pas la même réalité que celui d'écrivain. La reconnaissance de l'auteur va pourtant se fonder sur cette stratégie.

Toujours à propos de ces stratégies, Casanova montre comment elles peuvent être historiquement datées. Elle prend notamment l'exemple des tentatives de transposition des pratiques orales à l'écrit comme autant de "moyens pour créer de la littérature $»^{12}$ et précise qu'elles s'effectuent préférentiellement grâce aux genres du conte et du théâtre. Sans réduire la production de Maillet à ces deux genres, on peut dire qu'elle se positionne par rapport à ceux-ci à travers le statut qu'elle revendique.

9. Manon Laparra, «Parole manifeste : le cas de la littérature acadienne moderne », Revue de littérature comparée, 2006, n³17, p. 72.

10. Benoît Denis et Jean-Marie Klinkenberg, La Littérature belge - Précis d'histoire sociale, Bruxelles, Éditions Labor, 2005, p. 33.

11. Pascale Casanova, La République mondiale des Lettres, Paris, Seuil, 1999, p. 246.

12. Id., p. 309. 


\section{La place de la conteuse dans le discours de Maillet}

Dans divers entretiens - qu'elle a accordés à la critique tout au long de sa carrière -, Antonine Maillet a revendiqué la place importante de l'oralité dans son écriture. L'oscillation entre oral et écrit n'en est pas moins complexe pour autant. Cette articulation mobilise en effet le statut d'écrivaine/conteuse - comme nous l'avons dit - mais aussi la question d'une langue teintée d'oralité.

Maillet assume clairement ces composantes: "Je me dis que je suis probablement la dernière d'une race, celles des conteurs. Je me situe à la jointure de la tradition orale et de l'écriture. ${ }^{13} \mathrm{Au}$ sujet de sa langue d'écriture, elle dit également ceci : "Je n'invente pas les mots. Je n'invente rien. Je cherche à réaliser la transposition écrite d'une chose entendue. Ce n'est pas une langue phonétique passée dans l'écrit, mais un récit raconté par écrit... J'écris... avec accent. » ${ }^{14}$

Dans la première de ces deux citations, il est intéressant de remarquer que Maillet souligne non seulement l'oralité de ses écrits, mais aussi l'appartenance à toute une tradition orale et, au-delà, à la tradition populaire acadienne. Au détour d'une ligne, nous pouvons apercevoir ce que Maingueneau appelle la « vocation énonciative $»^{15}$. Maillet, en s'inscrivant dans la lignée des conteurs - cette " race » à laquelle elle fait allusion légitime en quelque sorte son projet d'écriture en attirant l'attention sur une tradition culturelle particulière et méconnue au-delà de la sphère acadienne. Elle envisage également la spécificité de sa position par le terme " jointure », ce qui renvoie à la transition entre oral et écrit évoquée avec Casanova ${ }^{16}$. Si le terme " jointure » apparaît important, c'est qu'il désigne aussi le versant écrit attaché à sa production. La filiation revendiquée se fait aussi vis-à-vis d'une certaine tradition littéraire, dont une des composantes les plus visibles est sans doute l'intertextualité rabelaisienne. Cette composante mérite d'être évoquée car nous pouvons noter, avec Maingueneau, qu'il existe une "relation étroite entre le positionnement, la mémoire intertextuelle et l'investissement de tel ou tel genre ${ }^{17}$. C'est sans doute à ce niveau qu'il peut être intéressant de rapprocher la perspective de Maingueneau et l'analyse du discours critique. Les concepts de Maingueneau permettent de mettre au jour la cohérence des différents

13. Antonine Maillet citée dans René LeBlanc, « Antonine Maillet conteuse de l'Acadie », Québec français, 1985, n60, p. 36.

14. Antonine Maillet citée dans Madeleine Ducrocq-Poirier, «Perception et réception en France d'Antonine Maillet et de son œuvre », dans Marguerite Maillet et Judith Hamel (dir.), op. cit., p. 53.

15. Dominique Maingueneau, op. cit., p. 119.

16. Pascale Casanova, op. cit., p. 309.

17. Dominique Maingueneau, op. cit., p. 130. 
éléments qui composent l'énonciation littéraire. En associant cette perspective à l'analyse du discours critique, on peut s'interroger sur la façon dont ressortent certains phénomènes et de quelle manière ils sont perçus d'une sphère culturelle à une autre.

\section{Une réception critique plurielle}

\section{La sphère québécoise}

Si l'on examine dans la sphère québécoise les réactions de la critique, on remarque une perception plutôt négative de la littérarité des textes et en particulier de l'intertexte rabelaisien - qui a contribué au succès en France de l'écrivaine. Jeanne Demers, qui s'intéresse à la réception du Huitième Jour ${ }^{18}$ dans la presse québécoise, prend l'exemple d'un article intitulé "Le rabelaisisme a encore frappé »19, témoignant d'une perception assez négative du texte et de l'usure du procédé. La critique poursuit en associant à celui-ci un second, Don l'Orignal, avant d'émettre l'hypothèse qu'ils sont "trop "écrits" peut-être, trop riches en intertextes littéraires en dépit de leurs évidentes racines folkloriques ${ }^{20}$. C'est la composante folklorique qui retient l'attention de la critique - en dehors de l'oralité - au point qu'elle va faire l'objet de rapprochements. Donald Smith déclare à titre d'exemple : "Jacques Ferron a écrit les "contes du pays incertain" inspirés du folklore gaspésien et beauceron : Antonine Maillet reproduit les contes et légendes d'une Acadie qui s'éveille. $»^{21}$

La place faite par la critique aux procédés d'écriture semble mince. Le travail d'écriture en soi ne transparaît pas, le caractère « écrit » se révélant forcé ou au contraire superficiel. Il s'agit de "transposition » ou de « transcription »: "L'œuvre entière n'est que la transcription imprimée de faits qu'on apprend d'un raconteur ou de la raconteuse-auteur. " ${ }^{22}$ Cette tendance s'explique selon Demers par l'« extraordinaire sens de la parole [de Maillet]. Une parole que journalistes et critiques n'osent transformer en réflexion personnelle et qu'ils rapportent toute chaude et vibrante, plus souvent qu'autrement au détriment de l'œuvre sur laquelle ils se trouvent mettre une sourdine ${ }^{23}$. Il existe donc une certaine coïncidence entre le discours de l'auteur et l'attitude générale de la critique.

18. Antonine Maillet, Le Huitième Jour, Montréal, Leméac, 1986.

19. Jeanne Demers, «Une entreprise de séduction ou la réception régionale au Québec d’Antonine Maillet », dans Marguerite Maillet et Judith Hamel (dir.), op. cit., p. 307.

20. Id., p. 298.

21. Donald Smith, "L'Acadie, pays de la ruse et du conte », dans les Lettres québécoises, $1980, \mathrm{n}^{\circ} 19, \mathrm{p} .45$.

22. René LeBlanc, "L'oralité du style dans les romans d'Antonine Maillet », Revue d'histoire littéraire du Québec et du Canada français, 1986, n 12, p. 58.

23. Jeanne Demers, op. cit., p. 311. 
Le discours mailletien oriente donc l'interprétation de l'œuvre et sa réception tout en véhiculant une vision particulière du fait littéraire. Demers évoque d'ailleurs le rôle actif joué par l'auteur dans ce processus de réception :

S’il s'agissait [...] d'une très lucide entreprise de séduction établie sur le modèle même de la relation conteur-auditoire? Madame Maillet jouant à Jour sans nom avec le temps, hypercodant son discours quotidien et trichant sur le « je » narrateur au point de se confondre avec lui... ${ }^{24}$

Pour revenir sur la manière dont la critique québécoise traite la dimension folklorique des textes, Donald Smith établit, par exemple, une comparaison avec Jacques Ferron, qui semble isoler davantage la production de Maillet pour mieux la définir. C'est dans ce sens qu'opère une partie de la critique, qui ne perçoit pas le folklore comme négatif. Raymond Pagé indique à ce sujet que "plusieurs critiques ne s'offusquent pas de cette atmosphère tissée de couleur locale, de musique du pays, cette galerie pittoresque de portraits [...] $»^{25}$. Mais d'autres réactions existent, dont celle de Robert Lévesque notamment, qui considère ce trait comme complaisant voire "réactionnaire » et lié à une certaine vision "conservatrice et mythique $»^{26}$.

Le caractère ambigu du discours critique est particulièrement sensible si l'on considère la réception de la Sagouine. Au cœur de cette réception se pose en effet la question de l'appartenance du texte au théâtre québécois, comme le montre Pagé : "Michel Vaïs [...] classait La Sagouine comme une "des pièces mythiques de notre répertoire, inévitables pour quelque étranger qui voudrait comprendre le Québec". " ${ }^{27}$ Cette tendance à l'assimilation qui fonctionne de pair avec un certain discours sur l'universalité du personnage, s'explique, d'après le critique, par le fait que le personnage de la Sagouine est un « exemple pour un peuple et un théâtre québécois aux prises avec le ressac des événements d'octobre $1970{ }^{28}$. Le critique poursuit ensuite en montrant la mutation de ce discours dans le temps :

Les instances de productions ont récupéré la parole dénonciatrice pour en faire une représentation annuelle,

24. Id., p. 299.

25. Raymond Pagé, "Spectateurs et lecteurs des œuvres d'Antonine Maillet », dans Marguerite Maillet et Judith Hamel (dir.), op. cit., p. 322.

26. Robert Lévesque cité dans Raymond Pagé, op. cit., p. 322.

27. Raymond Pagé, op. cit., p. 316.

28. Id., p. 323. 
donc une tradition [...] les mouvements de rejet ont surgi des instances de légitimation. Un rejet fondé sur la crainte de voir ressurgir les spectres d'Évangéline et de Maria Chapdelaine, main dans la main, au cœur du pays à faire. ${ }^{29}$

Pour ce qui est des pièces postérieures, le folklore devient de plus en plus l'objet d'attaques. Certains critiques "accusent [Maillet] de camoufler l'appel à la différence et à la lutte des classes sous un vêtement de folklore [...], un exotisme qui donne bonne conscience [...] ${ }^{30}$. Au passage, nous pouvons remarquer que la lecture de Maillet se fait volontiers, dans la sphère québécoise, idéologique et politique.

L'exotisme, lié étroitement à la composante folklorique des textes, est un élément qui recoupe de nouveau les propos de l'auteur qui déclarait en 1978 : "Si dans dix ans c'est fini et que plus personne ne s'intéresse à ce que je fais, cela n'aura été qu'une espèce de vague d'exotisme. $»^{31}$

\section{La sphère française}

L'exotisme est également un motif récurrent dans la critique française et ce, au-delà du prix Goncourt, comme le montre Robert Mane, en citant cet extrait de la quatrième de couverture de La Gribouille ${ }^{32}$ : "Après Pélagie-la-Charette, prix Goncourt 1979, Antonine Maillet [...] fait revivre tout un monde foisonnant, facétieux, babillard, gai comme la vie, simple comme la terre, et vrai, et jeune, comme nous ne savons plus l'être dans les "vieux pays". »33

Pour s'approcher de ce type de discours, considérer le rapport de l'œuvre de Maillet à l'espace français s'avère fondamental. Ce rapport passe en particulier par le concept de francophonie, comme l'indique Robert Mane. Le critique tend à démontrer qu'une des variables qui auraient orienté le choix du jury Goncourt en faveur de Maillet est l'idée même de francophonie, ou du moins son utilisation : "Le français lointain [...] ce surgissement et ce foisonnement littéraire d'une francophonie lointaine [...] en un moment où le français ne va pas si bien dans le monde. "34 L'idée de défense de la langue française - en tant que préoccupation francofrançaise - revient de manière récurrente. C'est le cas, par exemple, de Jean-Paul Morin : "Les Goncourt récompensent un des plus solides défenseurs de la langue française hors de l'Hexagone. À l'heure où l'on se doit,

29. Id., p. 323.

30. Id., p. 318.

31. Antonine Maillet citée dans Raymond Pagé, op. cit., p. 313.

32. Antonine Maillet, La Gribouille, Paris, édition Livre de poche, 1984.

33. Robert Mane, "Pélagie-la-Charrette en France », dans Marguerite Maillet et Judith Hamel (dir.), op. cit., p. 44.

34. André Stil cité dans Marguerite Maillet et Judith Hamel (dir.), op. cit., p. 41. 
de langue ferme de lutter, même ici, contre les néologismes anglo-saxons, le combat d'Antonine Maillet l'Acadienne devient exemplaire. "35 Robert Mane indique, avec justesse d'ailleurs, ce qui se cache derrière cet usage de la notion de francophonie : "en même temps Maillet nous apportait le meilleur des messages, celui de notre grandeur culturelle plus rayonnante, plus ferme que jamais, par la grâce de la francophonie ${ }^{36}$.

Bien que cette dimension de la réception critique ne se retrouve pas dans la sphère québécoise, il existe des parallèles entre les deux ensembles. Un de ceux-ci est identifié explicitement par Mane : "Alors prend corps un phénomène dont nous n'allons cesser de percevoir les effets jusqu'à - et y abondamment comprise - la réception de Pélagie-la-Charrette; il s'agit du contraste entre la réception de l'écrit et de ce que nous appellerons pour l'instant l'oral et le visuel. „37 Cette citation est intéressante, car elle renvoie non pas à l'oralité comme composante textuelle, mais au phénomène médiatique Maillet.

Cette médiatisation débute dès septembre 1977 avec l'émission "Apostrophes » de Bernard Pivot. Mane met d'ailleurs en relation la création du personnage médiatique et l'obtention du Goncourt : "L'obstacle de l'écrit s'en trouvant oblitéré, des millions de téléspectateurs peuvent décréter que le prochain prix Goncourt appartient à cette superbe parleuse. "38 $^{38}$ De nouveau, nous retrouvons le discours mailletien comme un des éléments marquant le processus de légitimation et de reconnaissance du travail de l'écrivaine.

Lorsque l'on envisage le discours critique québécois (à commencer par le plus favorable), on peut remarquer la superficialité avec laquelle les textes sont abordés. Cette superficialité caractérise aussi la critique française : "Antonine Maillet est à l'Acadie ce que Jean Giono fut à la Provence et Ramuz au Valais. »39

Mais cet extrait témoigne également du caractère régional associé à l'œuvre de Maillet. À ce propos, Madeleine Ducrocq-Poirier constate que pour « la majorité des Français [...] Antonine Maillet est ni plus ni moins qu'un écrivain régionaliste de ce que l'on appelait, il n'y a pas si longtemps

35. Jean-Paul Morin cité dans Marguerite Maillet et Judith Hamel (dir.), op. cit., p. 42.

36. Robert Mane, op. cit., p. 42.

37. Id., p. 33 .

38. Id., p. 35 .

39. Jacques Cellard cité dans Robert Mane, op. cit., p. 36-37. 
encore, le Canada français $4^{40} »^{41}$. Mais une fois cette division révolue, l'appartenance de l'écrivaine apparaît comme problématique. Ducrocq-Poirier déclare d'ailleurs que "curieusement, alors qu'elle ne cesse d'évoquer l'Acadie à tous moments, les Français la perçoivent difficilement comme un écrivain acadien spécifique. Écrivain francophone d'Amérique du Nord, certes, écrivain canadien, bien sûr, mais d'Acadie, qu'est-ce à dire? Les références font alors défaut ${ }^{42}$. Cela prouve que le discours mailletien véhiculé par les médias - fonctionne particulièrement bien. Toutefois, identifier l'écrivaine à une sphère littéraire précise est plus difficile en raison du faible degré d'institutionnalisation du champ et des relations complexes qu'il entretient avec son voisin québécois. Cette situation est renforcée par l'usage du conte :

Chez les lecteurs français, un peu au fait de la littérature québécoise et de ses traditions orales, un rapprochement était spontanément établi entre la fantastique odyssée de Pélagie [...] avec les aventures au long cours de ceux qui couraient la chasse-galerie dans les contes québécois. Même atmosphère quasi surhumaine et même agrandissement épique des événements et des personnages... ${ }^{43}$

Une certaine confusion règne tant au niveau territorial qu'identitaire et les rapprochements effectués sont parfois de l'ordre du cliché comme en témoigne "l'expression française usée jusqu'à la corde de "lointains cousins de l'autre côté de l'Atlantique" "44, comme le souligne Ducrocq-Poirier. Cette confusion est aussi relayée par la situation de l'auteur à Montréal - due en particulier au très faible degré d'institutionnalisation du champ acadien à l'époque de ses « débuts ».

\section{La sphère acadienne}

James de Finney décèle dans la presse locale et notamment dans le journal L'Évangéline certains phénomènes qui contrastent avec la réception québécoise ou française. En ce qui concerne l'exploitation par

40. "Le terme "Canada français", apparu à la suite de l'adoption du pacte confédératif fondant les États du Canada (1867), servait à l'origine à désigner la communauté francophone de ce pays, sans rattachement géographique bien délimité. Depuis la fin des années 1960, il sert à nommer l'ensemble des francophones répartis sur le territoire canadien, le plus souvent à l'exception des habitants de la province du Québec » (Paul Aron, Denis Saint-Jacques et Alain Viala (dir.), Le Dictionnaire $d u$ littéraire, Paris, Puf, 2004, p. 74).

41. Madeleine Ducrocq-Poirier, op. cit., p. 49.

42. Id., p. 48.

43. Id., p. 49.

44. Id., p. 49. 
les médias du personnage Maillet en Acadie, ce phénomène débute de manière plus précoce et «se traduit concrètement par les manchettes de journaux, par le rituel des lancements, des interviews, des prix littéraires, etc. dont L'Évangéline fait abondamment état. Parfois, cette médiatisation donne lieu à une espèce de connivence tacite entre l'auteur et les journalistes ${ }^{45}$. Mais cet intérêt médiatique va s'interrompre de façon précoce après l'événement du Goncourt. La disparition de Maillet des lignes du journal - dont la parution prend fin en 1982 - s'expliquerait par cette surexploitation médiatique même.

Mais la grande différence se trouve au niveau de l'appropriation « $\mathrm{col}$ lective de l'auteur, des textes et même de la gloire qui en a découlé ${ }^{46}$, comme le note de Finney. La composante identitaire prédomine par la lecture des composantes sociale, politique, économique ou encore linguistique. Quant à l'approche esthétique, elle " est fondée avant tout sur les fonctions expressive et référentielle du langage ${ }^{47}$. Le discours mailletien n'est pas étranger non plus à ce phénomène puisque "l'auteur a d'ailleurs encouragé cette attitude par la conception qu'elle a de son rôle : le "conteur écrivain", explique-t-elle, "garde un pied dans la tradition et la collectivité; alors il a l'impression de se faire le porte-parole de cette collectivité" ${ }^{48}$.

En se proposant de représenter la collectivité acadienne, Maillet en a surtout produit une vision particulière, sur laquelle nous reviendrons. Avant cela, nous nous interrogerons sur l'emploi d'une certaine langue qu'implique le rôle de porte-parole.

\section{La question de la langue}

La question de la langue n'est apparue pour l'instant qu'en filigrane. Mais, en abordant la sphère acadienne, cette question devient centrale, dans la mesure où elle est liée à la problématique identitaire. Judith C. Perron s'est interrogée à ce propos : «comment le spectateur/spectatrice perçoit-il/elle la langue de la Sagouine et quelle fonction lui accorde-t-il) elle dans la société? "49

Dans la sphère acadienne, les positions varient. Certains condamnent cette langue, car elle apparaît comme non représentative : «les expressions [sont] baroques, choquantes, humiliantes [...] quelques-unes n'ont

45. James De Finney, "Antonine Maillet : un exemple de réception littéraire régionale », Revue d'histoire littéraire du Québec et du Canada français, 1986, n 12, p. 21.

46. Id., p. 22.

47. Id., p. 26.

48. Id., p. 22.

49. Judith C. Perron, « La réception de la Sagouine : tétraglossie et fonction de création sociale », dans Marguerite Maillet et Judith Hamel (dir.), op. cit., p. 236. 
jamais existé et [...] le reste nous rabaisse plutôt que de nous rehausser dans l'opinion de grand nombre de gens bien pensants "50. La question du rapport à une certaine norme linguistique apparaît dès lors comme fondamentale pour comprendre ce type de réactions.

Mais cette position contraste avec la perception de la langue de La Sagouine comme un bien collectif : "son langage est de l'Acadien pur, tel qu'il a été transmis de père en fils $»^{51}$. Même si le caractère représentatif associé à la langue n'est pas unanime, la dimension identitaire devient prépondérante y compris dans les critiques davantage négatives de la langue de La Sagouine. Cela explique également les caractéristiques propres à la sphère acadienne vis-à-vis des autres ensembles étudiés précédemment.

Pour illustrer cette différence, si l'on revient sur la sphère française, la représentation de la langue apparaît cette fois dans une certaine indétermination. Ducrocq-Poirier, face à l'impossibilité de la critique à décrire cette langue, arrive d'ailleurs à ce constat : «le public français, toujours pas mieux informé en la matière, se demande aujourd'hui encore s'il existe un français acadien, comme il existe un français québécois et en quoi il se distingue foncièrement de ce dernier [...] $»^{52}$.

Quant à la sphère québécoise, une certaine valeur mythique est associée à cette langue, "surtout lorsque la critique fait appel au caractère primitif de la langue ${ }{ }^{53}$, comme le note Perron. La perception de cette langue en tant que "vernaculaire », note la critique, n'a plus cette valeur identitaire présente dans la sphère acadienne. La composante identitaire apparaît comme étrangère à la sphère proprement québécoise.

La question de la langue d'écriture de Maillet nous semble dès lors dépasser la simple transposition de l'oral à l'écrit. Elle suscite des perceptions très diverses au sein de la sphère acadienne, mais aussi lorsque l'on considère les autres ensembles. Cette multiplicité des points de vue s'explique par l'ancrage de ces regards dans un espace spécifique qui conditionne dans une certaine mesure les représentations.

Cependant, il faut également noter que la langue mailletienne se présente comme une construction. Maingueneau insiste d'ailleurs sur cette caractéristique du texte littéraire :

L'auteur ne place pas plus son œuvre dans une langue que dans un genre. Il n'y a pas d'un côté des contenus, de l'autre

50. Henri Richard cité dans Marguerite Maillet et Judith Hamel (dir.), op. cit., p. 241.

51. Melvin Gallant et Pierre-André Arcand cités dans Marguerite Maillet et Judith Hamel (dir.), op. cit., p. 242.

52. Madeleine Ducrocq-Poirier, op. cit., p. 53.

53. Judith C. Perron, op. cit., p. 239. 
une langue déjà donnée qui permettrait de les véhiculer, mais la manière dont une œuvre gère « l'interlangue » est une dimension constitutive de cette œuvre. 54

Cette gestion s'effectue chez Maillet dans l'oscillation entre oralité et littérarité, qui crée une certaine tension dans ses textes et renvoie, audelà, à l'ambiguïté du statut de l'auteur. Dans un bref parallèle à Rabelais, nous retrouvons cette idée de construction langagière :

\section{[...] comme Lise Gauvin l'a rappelé, la langue de Maillet, comme celle de Rabelais, est une langue «mi-réelle, mi-rêvée » ou encore, selon Pierre l'Hérault, un français qui n'est surtout pas parlé à Bouctouche et, dans le cas de Rabelais, surtout pas à Chinon. 55}

Ce rapprochement avec la langue de Rabelais n'est d'ailleurs pas anodin, comme nous l'avons vu, puisqu'il renvoie à une stratégie de légitimation de l'écrivaine. Cette filiation permet à Maillet de revendiquer une certaine pureté du français acadien en le rapportant à une variété consacrée de la langue française, à savoir la langue littéraire, incarnée par Rabelais. Le discours de l'écrivaine se révèle dès lors ambivalent puisque, s'il prône la spécificité de la variété acadienne, c'est en s’inscrivant dans le sillage des discours normatif et puriste sur la langue française.

La perception de la langue et des textes et de l'auteur s'avère donc plurielle, comme nous l'avons montré. Toutefois, l'idée de Maillet porteparole, "conteuse ", de l'Acadie traverse l'imaginaire collectif et cela, toutes sphères confondues, notamment au travers de son image médiatique. Nous envisagerons maintenant les textes en observant de quelle manière ils participent au positionnement de l'écrivaine en accordant un intérêt particulier à la vision de l’Acadie qu'ils suggèrent.

\section{Entre scénographie et paratopie, une énonciation spécifique}

Le statut de l'écrivaine conteuse ne se retrouve pas seulement dans le discours critique ou celui de l'auteur, mais aussi dans les textes de Maillet. Nous tenterons grâce aux concepts de "paratopie » et de "scénographie » développés par Maingueneau ${ }^{56}$ d'approcher le discours

54. Dominique Maingueneau, op. cit., p. 139.

55. Claude La Charité, «Antonine Maillet et Rabelais : contexte, originalité et fortune de sa thèse Rabelais et les traditions populaires en Acadie ", dans Marie-Linda Lord (dir.), Lire Antonine Maillet à travers le temps et l'espace, Moncton, Institut d'études acadiennes, 2010, p. 41.

56. Dominique Maingueneau, op. cit., p. 139. 
littéraire de Maillet, en particulier le positionnement de l'auteur qui se trouve au centre de son dispositif narratif.

L'auteur nous raconte en effet l'histoire fictive de personnagesconteurs au fil de leurs aventures et des générations qui se succèdent. Et ce mécanisme est mis en place dès le prologue de Pélagie-la-Charrette:

\section{[...] l'histoire continue.}

... Elle continue encore dans la bouche de mon cousin Louis à Bélonie, qui la tient de son père Bélonie à Louis, qui la tenait de son grand-père Bélonie - contemporain et adversaire de la Gribouille - qui l'avait reçue de père en fils de ce propre Bélonie, fils de Thaddée, fils de Bélonie premier qui, en 1770, fêtait ses nonante ans assis au fond de la charrette même de Pélagie, première du nom. ${ }^{57}$

Cette citation illustre la mise en scène de la parole qui traverse toute l'œuvre romanesque et a fortiori théâtrale de Maillet. L'écrivaine nous décrit moins les contes auxquels elle fait allusion qu'elle ne représente le passage de cette " histoire » dans les paroles de ses personnagesconteurs.

Le concept de "scénographie », en tant que "scène de parole que le discours présuppose pour pouvoir être énoncé et qu'en retour il doit valider à travers son énonciation même $»^{58}$, permet de montrer la cohérence du discours mailletien, qu'il évolue au niveau du texte littéraire ou en dehors de celui-ci.

L'écrivaine met en scène une parole mouvante qui se modifie de conteur en conteur au fil du temps et des interactions entre personnages. Le passage suivant est significatif à cet égard:

«Si parsonne l'a onques vue, comment c'est qu'on sait qu'elle est noire, votre charrette? Que planta Pélagie en plein dans le front du vieux radoteux.

$-\mathrm{Hi} ! »$

... pour toute réponse de Bélonie. Car en bon conteur de sa profession, il se réservait pour ses contes, Bélonie, et ne gaspillait jamais sa salive dans des obstinations perdues. ${ }^{59}$

57. Antonine Maillet, Pélagie-la-Charrette, op. cit., p. 11.

58. Dominique Maingueneau, op. cit., p. 192.

59. Antonine Maillet, Pélagie-la-Charrette, op. cit., p. 13. 
Le texte évolue dès lors dans un mouvement paradoxal. D'un côté, il exprime le mouvement d'une parole changeante et empreinte d'oralité mais, tout en la mettant en scène, il participe, de l'autre, à son figement dans l'écriture. Le statut de l'écrivaine/conteuse prend ici tout son sens puisqu'en s'inscrivant comme réceptacle de toute une tradition orale, celle des conteurs acadiens, Maillet devient, par son passage à l'écriture, symbole de sa fin. Ce phénomène est à associer avec la vision de la société acadienne que Maillet tente de donner par le prisme de la fiction. Cette dimension est particulièrement visible dans une triade de romans - Pélagie-la-Charrette ${ }^{60}$ (1979), Cent ans dans les bois ${ }^{61}$ (1981) et Pierre Bleu $^{62}$ (2006) - qui se distinguent par la perspective historique qu'ils véhiculent.

La scénographie mise en place par Maillet permet au lecteur d'accéder à toute une représentation de la société acadienne dans son évolution. Pélagie-la-Charrette traite du retour qui suit la déportation, Cent ans dans les bois est orienté sur la période suivante, la renaissance acadienne. Quant à Pierre Bleu, il couvre la période qui s'étend des années 1880 au lendemain des années 1970 . Bien que ce roman se rattache davantage à la production récente de l'auteur, comme l'a montré Robert Viau ${ }^{63}$, le critique l'envisage tout de même par sa perspective historique comme une suite de Cent ans dans les bois.

Au moment où l'œuvre de Maillet accède à une certaine reconnaissance et tend à fonder un des premiers maillons de la littérature acadienne moderne, le regard qu'offre l'auteur, en adéquation avec la scénographie que nous avons relevée, est tourné vers la passé.

Mais Maillet n'en apporte pas moins une certaine nouveauté dans le champ de la littérature acadienne. Comme l'a montré Denis Bourque en s'intéressant à "l'horizon d'attente du lecteur acadien des années soixante-dix ${ }^{64}$, Maillet modifie, via ses textes, certains schèmes présents dans l'imaginaire collectif. Elle s'oppose, pour ne prendre qu'un exemple, au mythe de L'Évangéline de Longfellow qui associait à cette figure mythique le peuple acadien dans sa résignation face à son propre martyr. Cependant, même si l'on peut noter cette rupture, il ne faut pas sous-estimer le rapport problématique au temps qu'entretient Maillet.

60. Antonine Maillet, Pélagie-la-Charrette, op. cit. (Paris, Grasset, 1979).

61. Antonine Maillet, Cent ans dans les bois, Montréal, Leméac, 1981.

62. Antonine Maillet, Pierre Bleu, Montréal-Paris, Leméac/Actes Sud, 2006.

63. Robert Viau, Antonine Maillet : 50 ans d'écriture, Ottawa, Éditions David, 2008.

64. Denis Bourque, "Horizon d'attente du lecteur acadien des années soixante-dix : dialogue avec le mythe » dans Marguerite Maillet et Judith Hamel (dir.), op. cit., p. 199-214. 
La notion de " paratopie ", que nous avons définie précédemment avec Maingueneau 65 , s'avère utile pour observer ce rapport paradoxal au temps. Au moment où son œuvre accède à une certaine reconnaissance, c'est-à-dire dans le courant des années 1970, l'espace-temps dans lequel Maillet inscrit son œuvre est celui de la fin du xix siècle, les années 1880 plus précisément. Cet ancrage temporel apparaît avec Bélonie à la fin de Pélagie-la-Charrette : "Le temps était beau en 1880, Bélonie lui-même le dit. Dépêchez-vous! II ne fallait point partir en retard encore un coup. Le pays avait un siècle à rattraper. " ${ }^{66}$ Dans Cent ans dans les bois, le récit se clôt avec Jérôme-le-menteux et la convention nationale de 1881. Il est d'ailleurs intéressant de remarquer que la narratrice s'inscrit explicitement dans cette époque comme l'extrait suivant le suggère : «...Et du ventre de ma grand-grand-mère, j'ai vu disparaître Jérôme à l'horizon. La fourmilière était toute grande ouverte. Et j'ai compris que les fourmis étaient sorties $d u$ bois. $"{ }^{67}$ La signature de l'auteur est également significative de ce décalage temporel : "Montréal, automne 1981 ... cent ans plus tard ${ }^{68}$.

En ce qui concerne Pierre Bleu, le décalage temporel relevé pour les deux romans précédents tend à se résorber. L'auteur semble vouloir rattraper le retard qu'elle évoquait dans Pélagie-la-Charrette. Elle tente de retracer en effet l'histoire de l'Acadie de 1880 jusqu'au moment de son énonciation. Toutefois, un décalage persiste toujours même dans ce texte contemporain (Pierre Bleu, 2006). Ce texte évoque surtout la société acadienne de la fin $x x^{e}$ et du début du $x x^{e}$ siècle et se clôt au lendemain des années 1970. C'est d'ailleurs à ce moment précis que se projette l'auteur en se dédoublant dans le personnage de Bibiane-la-jeune, qui la représente dans la sphère diégétique. Ce procédé est récurrent dans la production de Maillet, où l'auteur se projette souvent dans certains personnages, comme celui de Radi.

Il est sans doute intéressant de souligner la coïncidence entre la première phase de production de l'auteur (années 1970), qui la fait connaître, et le climat de l'époque propice à une interrogation sur l'imaginaire acadien. Porter un regard sur l'histoire de la société acadienne traditionnelle jusqu'à son accession à la modernité se révèle important pour une société sur le point d'effectuer cette transition. L'identité collective ne se forge-telle pas, du moins en partie, en fonction des représentations du passé véhiculées d'une manière spécifique par ce type de littérature?

65. Dominique Maingueneau, op. cit., p. 52-53.

66. Antonine Maillet, Pélagie-la-Charrette, op. cit., p. 285.

67. Antonine Maillet, Cent ans dans les bois, op. cit., p. 354.

68. Id., p. 354 . 
L'idée même de collectivité était d'ailleurs au centre de la scénographie mise en place par l'auteur. L'espace traditionnel autour de l'âtre, qui réunit les conteurs autour des récits et paroles du passé, est d'ailleurs présent dans l'imaginaire des textes : "Et comme si cet aphorisme ne suffisait pas à convaincre le cercle des gicleux assis en demi-lune devant la maçoune [...]. " ${ }^{69}$ Cet espace de sociabilité peut être vu comme le lieu d'accès privilégié à une collectivité plus large : la société acadienne traditionnelle dans sa construction.

L'énonciation mailletienne se nourrit donc d'une tension paradoxale entre différentes temporalités, ce que nous pouvons rapprocher d'une certaine forme de " paratopie », que nous nommerons avec Maingueneau de type "temporelle $»^{70}$. Cela permet de comprendre, sans doute un peu mieux, la place qu'occupe Maillet dans l'institution littéraire et son rôle de transition. La dimension temporelle n'est toutefois qu'une dimension de la paratopie qui s'exprime de manières variées chez Maillet.

Comme le terme "paratopie » le suggère, l'énonciation littéraire se situe dans une "une localité paradoxale [...] qui n'est pas l'absence de tout lieu, mais une difficile négociation entre le lieu et le non-lieu $»^{71}$. Tout comme il a été question de " paratopie temporelle », nous pouvons observer la dimension "sociale » et "spatiale » de ce phénomène, comme l'indique Maingueneau ${ }^{72}$.

Différents textes de Maillet mettent en scène la confrontation entre deux groupes de personnes au sein d'une collectivité. C'est le cas notamment dans Les Crasseux ${ }^{73}$, où il s'agit d'une confrontation entre les gens "d'en haut » et les gens "d'en bas », dont les territoires sont séparés par une barrière - la séparation spatiale symbolisant la fracture sociale entre riches et pauvres. Mais c'est au théâtre que cette tension sociale s'incarne le plus clairement, dans le personnage type de la Sagouine. Son discours de dénonciation, parfois empreint de misérabilisme est étroitement lié à la condition du personnage occupé à " forbir »74 les biens des plus riches. Elle occupe par sa fonction la position paradoxale d'une pauvre chez les riches, ce qui lui permet de tenir un discours particulier: "Chaque affaire à sa place, que je vous dis, et une place pour chaque affaire. Ben icitte, y a pas de spitoune... ils prétendont que c'est pus la mode. Ça fait qu'ils mettont leux tchas de gomme dans la place, pis leu cendre partout [...]. „75

69. Antonine Maillet, Pélagie-la-Charrette, op. cit., p. 10.

70. Dominique Maingueneau, op. cit., p. 86.

71. Id., p. 52-53.

72. Id., p. 86.

73. Antonine Maillet, Les Crasseux, Montréal, Holt, Rinehart et Winston, 1968.

74. Antonine Maillet, La Sagouine - Pièce pour une femme seule, op. cit., p. 13.

75. Id., p. 13. 
Au-delà du renversement parodique présent dans cet extrait, les riches devenant en quelque sorte les vrais " crasseux », Maillet incarne avec $L a$ Sagouine l'inégalité sociale au sein d'une même société. Le mouvement de dénonciation et de critique que Maillet institue n'en est pas moins une tentative de définition et de consolidation d'un espace de parole où une société affirme son existence.

Le personnage d'Évangéline Deusse participe également à cette définition de l'Acadie, mais par un autre moyen, l'expression de la distance, du déracinement. II s'exprime en particulier par le sapin que l'exilée veut « enraciner » dans un parc de Montréal :

- Je peux pas ouère quoi c'est qu'ils avont contre les sapins.

T'es peut-être point un âbre des ville [sic], ben ... moi non plus

j'suis point une femme des villes et m'y v'la rendue asteur.

Ça fait qu'i' me r'semble que si i' me recevont icitte, faut ben qu'ils receviont itou un petit brin de la senteur et de la fraîcheur du pays. ${ }^{76}$

Il est difficile de ne pas faire le rapprochement entre cet extrait et la situation de l'écrivaine à Montréal, qui s'est éloignée de l'Acadie. Notamment en raison du très faible degré d'institutionnalisation du champ littéraire, ce choix s'explique en partie par la faiblesse du réseau éditorial acadien. Mais, lorsqu'on analyse les différents types de paratopie - spatiale, temporelle ou encore sociale - qui s'expriment au travers des textes de Maillet, on s'aperçoit de la cohérence de son discours. Le positionnement de Maillet s'enracine jusque dans ses textes, par lesquels elle tente de retracer la structuration de la société acadienne traditionnelle. C'est dans cette mesure que l'on peut, selon nous, parler d'œuvre charnière dans l'histoire des lettres acadiennes. Maillet signe en effet l'avènement d'une modernité littéraire qui se positionnera par rapport à sa production.

\section{Conclusion}

À la croisée de différents discours, nous avons cherché à indiquer quels étaient les enjeux liés au positionnement de l'auteur. Le statut d'écrivaine/conteuse, appuyé par le personnage médiatique de Maillet, a marqué et influencé la réception des œuvres de manière déterminante. Nous nous sommes attaché à étudier les mutations du discours critique en fonction de la sphère dans laquelle elles s'inscrivaient. Cela nous a permis de mieux comprendre les caractéristiques de ces ensembles par les relations qu'ils entretiennent entre eux. Et c'est sous cet éclairage qu'il est dès lors possible de montrer de quelle manière le dispositif narratif

76. Antonine Maillet, Évangéline Deusse, Montréal, Leméac, 1975, p. 23. 
élaboré par Maillet construisait l'espace de son propre déploiement. Dans ce mouvement, nous avons tenté d'indiquer de quelle façon le texte s'inscrit dans un univers social à un moment précis. La période durant laquelle la production de Maillet accède à une certaine reconnaissance est en effet significative au niveau tant littéraire que sociétal. Avec Maillet, la société acadienne traditionnelle se voit redéfinie et peut dès lors amorcer son passage à la modernité. 